Sancho-Álvarez, C., Jornet, J. M. y González-Such, J. (2016). El constructo Valor Social Subjetivo de la Educación: validación cruzada entre profesorado de escuela y universidad. Revista de Investigación Educativa, 34(2), 329-350. DOI: http://dx.doi.org/10.6018/rie.34.2.226131

\title{
El constructo Valor Social Subjetivo de la Educación: validación cruzada entre profesorado de escuela y universidad*
}

\author{
The Subjective Social Value of Education Construct: Cross- \\ Validation between School and University Teachers
}

\author{
Carlos Sancho-Álvarez, Jesús M. Jornet y José González-Such \\ Departamento de Métodos de Investigación y Diagnóstico en Educación. \\ Facultad de Filosofía y Ciencias de la Educación. Universitat de València. España.
}

\begin{abstract}
Resumen
La Educación posee un valor social evidente que puede presentarse mediante indicadores de evaluación socio-educativos. Si bien la relación entre contexto escolar y rendimiento es evidente en diversos países, esta relación va perdiendo consistencia en los últimos años; ya que aparecen sectores estudiantiles que superan las predicciones académicas esperadas. Para ello, se estudia el Valor Social Subjetivo de la Educación en función de dos grupos docentes -escuela y universidad-. La metodología es complementaria a través de comités de expertos/as y validación cruzada de constructo. Se realizan análisis sobre dos escalas -relevancia y susceptibilidad de cambio-y entre dos muestras diferenciadas para comprobar el consenso inter-subjetivo. Los datos muestran un acuerdo estadísticamente significativo en todas las dimensiones, sin embargo, se observan discrepancias dependiendo de la etapa escolar. Finalmente, se propone un constructo validado a través de cuatro dimensiones y trece indicadores de evaluación.

Palabras clave: evaluación educativa; validación de constructo; indicadores educativos; metodología de investigación.

Correspondencia: Carlos Sancho-Álvarez, Dpto. Mètodes d'Investigació i Diagnòstic en Educació. Facultat de Filosofia i Ciencies de l'Educació. Universitat de València. Avda. Blasco Ibañez, 30. 46010-Valencia. E-mail: carlos.sancho@uv.es

* Este trabajo se ha realizado en el marco del proyecto Sistema educativo y cohesión social: diseño de un modelo de evaluación de necesidades (SECS/EVALNEC). Ref. EDU2012-37437, financiado por el Ministerio de Economía y Competitividad de España. Asimismo, por "Ajudes per a la formació de personal investigador de caràcter predoctoral", en el marc del Subprograma Atracció de Talent 2013 del Vicerectorat d'Investigació i Política Científica de la Universitat de València.
\end{abstract}




\begin{abstract}
Education possesses an evident social value that can be shown by indicators of socioeducational assessments. While the relationship between educational context and performance is apparent in several countries, this relationship is losing consistency in recent years because the student-based sectors are appearing and outperforming the expected academic predictions. Therefore, the Subjective Social Value of Education is studied in two groups of teachers - school and university. The methodology is complementary through expert committees and construct cross-validation. Analyses are performed in two scales - relevance and sensitivity of change - and between two different groups to verify the inter-subjective consensus. The data shows a statistically significant agreement in all dimensions; however, discrepancies depending on the educational stage are observed. Finally, we propose a construct validated through four dimensions and thirteen assessment indicators.

Keywords: educational assessment; construct validity; educational indicators; research methodology.
\end{abstract}

\title{
Introducción
}

La percepción de la utilidad de asistir al colegio puede indicar la predisposición del alumno hacia el estudio, su grado de responsabilidad, su aprecio por el conocimiento, y sus expectativas sobre su trayectoria educativa futura. Todos ellos son atributos que, si bien se relacionan estrechamente con la dimensión cognitiva, pueden tener una explicación independiente (Krüger, Formichella \& Lekuona, 2015, p. 12).

En la actualidad, el Valor Social de la Educación (VSE) se manifiesta en determinadas ocasiones en relación a la visión de la propia sociedad en un mundo globalizado (Soriano, 2011). Después de haber sido mencionado como concepto en diferentes disciplinas y ámbitos de manera aislada (Casanova, 2012; García, 2012; Herrera, 2009), se plantea la necesidad de establecer un constructo de forma operativa y global para su posible evaluación desde el ámbito educativo (Jornet, Perales y Sánchez-Delgado, 2011).

En este sentido, se ha ido observando en las sociedades que la Educación presenta un claro valor social (Delors, 1996; Glewwe \& Kremer, 2006), que puede ser analizado a nivel objetivo -como expresión de lo que la sociedad invierte en la Educación y los resultados que tiene para el desarrollo personal y social- (Jornet, Sánchez-Delgado y Perales, 2014), al ser reflejo de la política educativa que se considere al respecto y de las consecuencias que ésta conlleva (Sancho-Álvarez, Jornet y Perales, 2013). En este sentido, después de algunos estudios en relación a posibles indicadores de evaluación, se encuentran algunas evidencias a nivel internacional pero siempre tratando el concepto de manera parcial (Sancho-Álvarez, 2013).

Además de asociar el rendimiento con la percepción escolar del alumnado, algo que también se continúa investigando son las diferencias de actitud que tiene el propio discente hacia la materia, en relación con el tipo de centro escolar en el que desarrolla su práctica educativa (García-García, Biencinto-López, Carpintero-Molina, Núñez-del-Río y Arteaga-Martínez, 2013). Asimismo, por ejemplo en el caso del valor general de las ciencias, las matemáticas y la lectura desde la percepción del alumnado (Organización 
para la Cooperación y el Desarrollo Económicos [OCDE], 2008), se puede observar en estudios internacionales la importancia de sacar buenas notas ante estas disciplinas académicas.

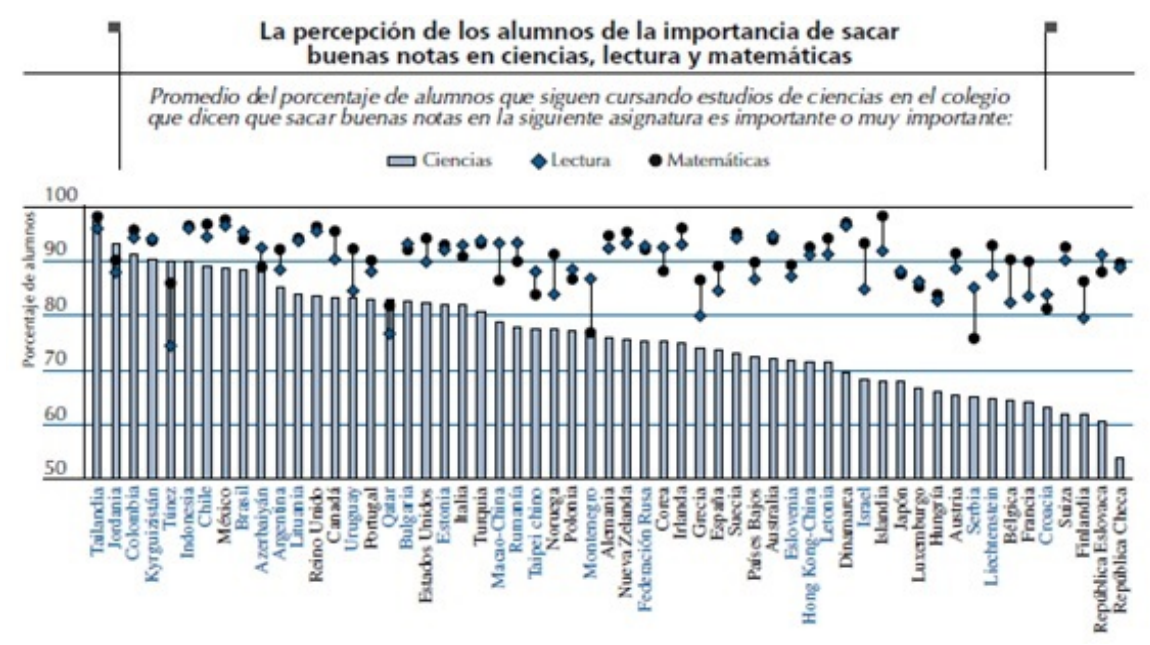

Gráfico 1. La importancia para el alumnado de sacar buenas notas (OCDE, 2008, p. 152).

Un elevado valor general de las ciencias puede reflejar aspectos positivos como la promoción de curso (Carstensen, Rost \& Prenzel, 2003). En este caso, también podemos observar información relevante acerca del propio valor general y personal de las ciencias mediante diferentes indicadores de evaluación.
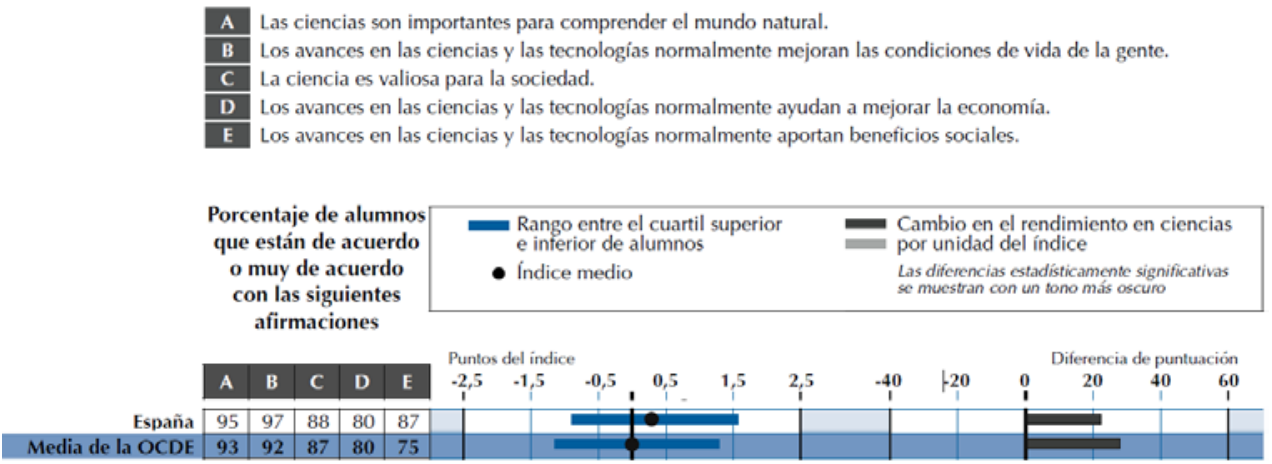

Gráfico 2. Índice del valor general de las ciencias (OCDE, 2006, p. 136)

Se puede comprobar como el caso español se sitúa por encima de la media -en 2006-, algo que actualmente ha podido variar debido a los cambios sociales y a la propia inestabilidad económica; aspectos que influyen directamente sobre la visión en general de la Educación (Ministerio de Educación, 2011). 
A Considero que las ciencias me ayudan a comprender lo que me rodea.

B Utilizaré las ciencias de muchas maneras cuando sea mayor.

C Algunos conceptos de las ciencias me ayudan a ver cómo me relaciono con los demás.

D Cuando termine el colegio tendré muchas oportunidades de utilizar las ciencias.

E Las ciencias son muy relevantes para mí.

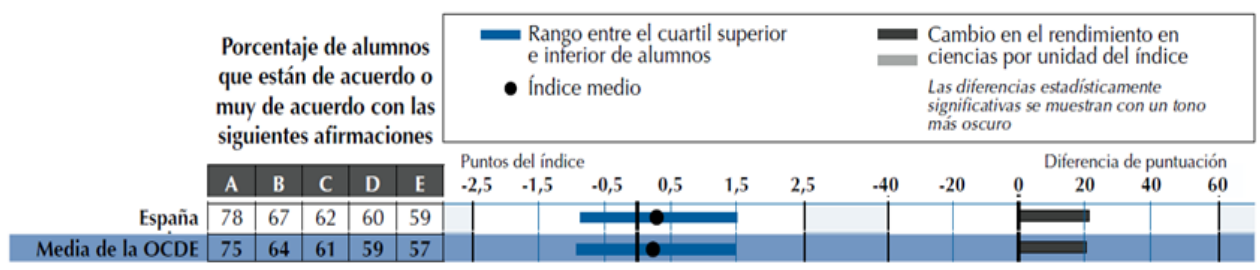

Gráfico 3. Índice del valor personal de las ciencias (OCDE, 2006, p. 139)

En esta línea, a lo largo de la historia ya desde el Informe Coleman de 1966 (Coleman, et al., 1966) y hasta la actualidad, se ha ido corroborando una estrecha relación entre el nivel sociocultural y el rendimiento académico del alumnado (Backhoff et al., 2008; Baer, 1999; Ruiz, 2009; Willms, 2006; Willms \& Somers, 2001). Por ello, es indispensable no sólo evaluar el aprendizaje de los estudiantes, sino también conocer las condiciones contextuales, en que éste ocurre, mediante cuestionarios de contexto (De la Orden, 2007; De la Orden y Jornet, 2012; Jornet, González-Such y Perales, 2012).

\section{Planteamiento del problema}

Si bien la relación entre los antecedentes de los estudiantes y el rendimiento escolar es evidente en diversos países, esta relación va perdiendo consistencia desde aproximadamente 2009, a través de los diferentes sistemas escolares (OCDE, 2010, 2011, 2012, 2013, 2014). Por lo tanto, es posible que el VSE pueda estar presente, en general, cuando una persona de un entorno desfavorecido, o en el otro extremo (niveles óptimos socio-económicos y culturales), desarrollan una tendencia contraria respecto a los resultados escolares que se esperarían según su nivel socio-económico cultural familiar (Jornet, 2010). En este caso, un claro ejemplo se da cuando el bienestar social es alto y se espera un nivel de logro académico también elevado, pero finalmente se produce un rendimiento escolar bajo; en contraposición a lo esperado según su nivel socioeconómico y cultural (Jornet, 2012). Asimismo, gran parte del alumnado de distintos países, que se encuentra en desventaja social y/o situaciones de dificultad, en muchos de los casos ha llegado a superar las predicciones académicas respecto a sus propios resultados escolares (OCDE, 2011, 2012).

Una mayor potencia económica regional puede derivar en un mayor esfuerzo inversor en educación. Sin embargo, salvo en lo que respecta a la calidad de las infraestructuras físicas, las diferencias en los recursos financieros, personales y materiales destinados a la educación no constituyen un factor relevante en la explicación de las diferencias de rendimiento entre comunidades del norte y del sur (Gil, 2014, p. 406). 


\section{Objetivos y contextualización}

En línea a una investigación más amplia (Sancho-Álvarez, 2013) y a partir de trabajos anteriores (Jornet et al., 2011; Sancho-Álvarez, et al., 2013; Sancho-Álvarez, Jornet y González-Such, 2014) se pretende realizar una validación cruzada de la dimensión subjetiva del constructo Valor Social de la Educación, en función de dos colectivos diferenciados de docentes -(1) profesorado de Infantil, Primaria y Secundaria; y (2) profesorado de Universidad-, para mejorar la propia formulación y poder establecer lineamientos de evaluación apropiados.

Ante el presente estado de la cuestión en sentido teórico-práctico, este estudio se enmarca dentro del modelo de evaluación para la Cohesión Social (Jornet, 2012; Jornet, Sancho-Álvarez y Sánchez-Delgado, 2014).

Tabla 1

Dimensiones del Modelo de evaluación para el desarrollo de la Cohesión Social a partir de la Educación

\begin{tabular}{|c|c|}
\hline Dimensiones & Constructos implicados \\
\hline Bienestar social (para todos) & $\begin{array}{l}\text { 1. Clima social y de aprendizaje en el aula } \\
\text { 2. Gestión social del aula } \\
\text { 3. Gestión de conflictos en el aula }\end{array}$ \\
\hline $\begin{array}{l}\text { Sostenibilidad (a lo largo de la } \\
\text { vida) }\end{array}$ & $\begin{array}{l}\text { 4. Competencias básicas (señaladas en el diseño curricular) } \\
\text { 5. Competencia y desarrollo emocional } \\
\text { 6. Valor social de la educación (VSE) } \\
\text { 7. Resiliencia }\end{array}$ \\
\hline $\begin{array}{l}\text { Equidad (en el acceso a recursos } \\
\text { y oportunidades) e integración de }\end{array}$ & $\begin{array}{l}\text { 8. Metodología didáctica (participativa, equipos, el estudiante } \\
\text { como protagonista de su aprendizaje...) }\end{array}$ \\
\hline la diversidad (personal y social) & $\begin{array}{l}\text { 9. Metodologia de evaluación (diversificada en métodos, } \\
\text { situaciones, tareas e instrumentos) } \\
\text { 10. Colegialidad docente } \\
\text { 11. Respeto y reconocimiento del otro } \\
\text { 12. Inclusividad: Atención a la diversidad fisica, cultural y/o social }\end{array}$ \\
\hline Participación (social) & $\begin{array}{l}\text { 13. Colaboración Familia-Profesorado-Escuela } \\
\text { 14. Estilos educativos familiares } \\
\text { 15. Estilos educativos docentes } \\
\text { 16. Sentido de pertenencia discente } \\
\text { 17. Responsabilidad social: Autoimagen del rol social respecto a } \\
\text { los entornos comunitarios (Escuela, Familia y Sociedad) }\end{array}$ \\
\hline
\end{tabular}

Nota: Adaptado de "La evaluación del impacto y la relevancia de la educación en la sociedad", En J. M. Jornet, P. Sánchez-Delgado, \& M. J. Perales, 2014. Valencia: PUV.

En la información expuesta se puede identificar un modelo educativo orientado hacia la promoción de la Cohesión Social desde la Educación. Para ello, se recoge una síntesis de las posibles dimensiones a tener en cuenta en la evaluación del alumnado, profesorado y familias. Dichas dimensiones se entiende que pueden establecerse como 
elementos de relación vertical entre diferentes planos de análisis: (1) aula, (2) escuela y (3) sistema (Jornet, 2012); así como, siendo conscientes de que la tarea educativa no englobaría todas las posibilidades de alcanzar la cohesión social en toda la sociedad, sino que habría que tener en cuenta también más disciplinas. Al respecto, el presente trabajo se centra únicamente sobre el constructo 6 -VSE-, recogido en dicho modelo en su segunda dimensión: Sostenibilidad (a lo largo de la vida).

Dentro de este marco conceptual, también es necesario operativizar el constructo en relación a otros modelos de evaluación que puedan identificar la relevancia y el impacto de la Educación en la sociedad. Así, como proponen Jornet et al. (2014) en el Modelo de Evaluación del Impacto y Relevancia de la Educación en la Sociedad (IRES), se puede observan referencias al VSE en la dimensión 3.

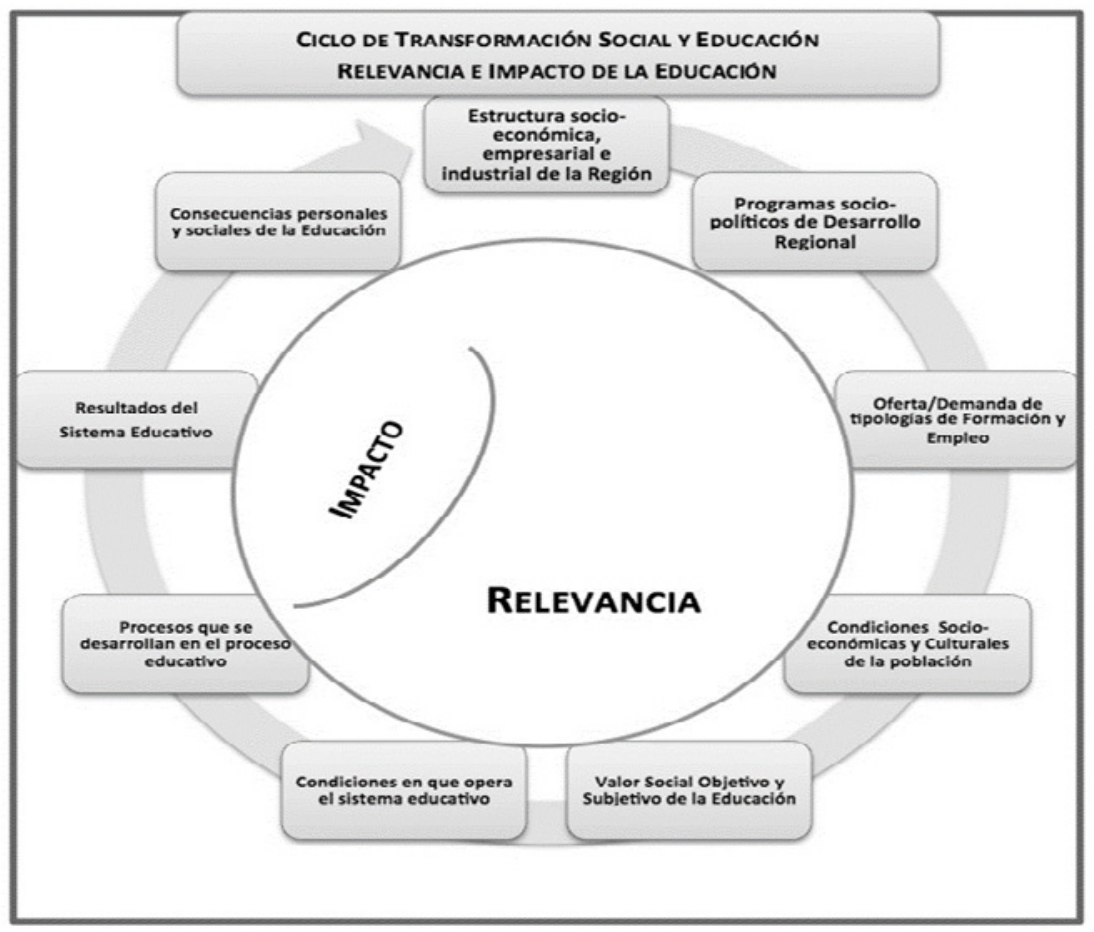

Figura 1. Modelo de Evaluación del Impacto y Relevancia de la Educación en la Sociedad -IRES- (Jornet et al., 2014).

\section{El concepto de Valor Social de la Educación (VSE)}

Cabe destacar que el VSE como constructo teórico, presenta dos facetas a considerar. Ya que puede ser analizado desde un prisma objetivo a través del Valor Social Objetivo de la Educación (VSE-Objetivo) -(1); referido a una serie de indicadores objetivos de evaluación en relación a las inversiones en Educación o las consecuencias de estar formado-. Y por otro lado, desde su faceta subjetiva mediante el Valor Social Subjetivo 
de la Educación (VSE-Subjetivo) -(2); por medio de indicadores complejos de evaluación sobre las percepciones personales y sociales de la población ante la importancia de la Educación-.

De acuerdo con Jornet et al. (2011) el VSE-Objetivo se refiere a:

(...) la utilidad que tiene la educación dentro de una sociedad para el desarrollo y la promoción de las personas en los ámbitos social y laboral, así como a las ventajas que aporte como elemento de prevención de la exclusión social, y como garantía para el desarrollo y la mejora de su bienestar a lo largo de la vida (p. 53).

Así como se considera el VSE-Subjetivo a:

(...) la percepción que los actores principales del proceso de enseñanza-aprendizaje (alumnado, familias y profesorado) tienen acerca de la importancia de la educación para la promoción social, laboral y del bienestar personal y colectivo, a lo largo de la vida (Jornet et al., 2011, p. 67).

En el presente trabajo, nos centraremos en su faceta subjetiva para dar respuesta a su aproximación conceptual y poder establecer una propuesta de indicadores de evaluación.

\section{Metodología}

\section{Diseño}

Se trata de un estudio que se sitúa metodológicamente en una posición de complementariedad cuantitativa y cualitativa (Bericat, 1998; Jornet, González-Such y Perales, 2013). Este enfoque metodológico se ha establecido por parte de nuestro grupo de investigación (GEM-Educo http://www.uv.es/gem/gemeduco), así como en el marco de referencia hacia el desarrollo de instrumentos de evaluación en los últimos años. Al respecto, se enfatiza la validación de constructo como un elemento de bondad sustantivo para cualquier instrumento de evaluación educativa. La atención prioritaria en la definición del constructo basándose en el consenso intersubjetivo para garantizar un planteamiento no arbitrario, ha supuesto un incremento de atención en los estudios basados en jueces, para poder superar las dificultades de aplicación de la validez y la ambigüedad en el significado (Cronbach, 1988), como sustento imprescindible para el diseño de instrumentos de medición y evaluación (Jornet y González-Such, 2009).

Los estudios mediante juicios de expertos/as, al centrarse en las inferencias y suposiciones del argumento específico e interpretativo de lo que se examina, ofrecen un argumento orientativo y detallado hacia el desarrollo de una validación eficaz (Kane, 2001). De acuerdo a estudios recientes en esta línea desarrollados a partir de análisis de validez de contenido, a través de jueces expertos se proponen modificaciones y mejoras muy valiosas hacia este tipo de procesos (Mérida, Serrano y Tabernero, 2015). 
El jueceo se ha estructurado en las siguientes fases, adaptando el modelo de diseño de instrumentos de evaluación de Jornet (1989) y Jornet y Suárez (1996) para la etapa de validación de constructo teórico: (1) comité inicial profesorado universitario expertos en medición/evaluación, (2) grupo de jueces profesorado infantil, primaria y secundaria, (3) grupo de jueces profesorado universitario y (4) comité final profesorado universitario expertos en medición/evaluación.

\section{Procedimiento}

Se ha desarrollado un comité de expertos/as inicial con profesorado universitario con el fin de discutir las dimensiones e indicadores de evaluación del constructo que se han propuesto en otras investigaciones (Sancho-Álvarez et al., 2014).

Tabla 2

Propuesta de constructo VSE-Subjetivo con resultados de validación previa

\begin{tabular}{|c|c|c|c|}
\hline \multicolumn{2}{|c|}{ DIMENSIÓN 1: EXPECTATIVAS Y METAS EDUCATIVAS } & \multirow{2}{*}{$\frac{\text { Relevancia }}{1}$} & \multirow{2}{*}{$\begin{array}{c}\text { Susceptibilida } \\
\text { d de cambio }\end{array}$} \\
\hline Indicador 1 & Nivel de aspiraciones educativas. & & \\
\hline Indicador 2 & $\begin{array}{l}\text { Expresión de las metas generales de logro que tiene el } \\
\text { alumnado, sus familias y el profesorado. }\end{array}$ & .95 & .90 \\
\hline Indicador 3 & Niveles a los que se aspira llegar, & 1 & .95 \\
\hline Indicador 4 & $\begin{array}{l}\text { Expectativas del profesorado y las familias sobre el } \\
\text { alumnado. }\end{array}$ & .90 & .95 \\
\hline \multicolumn{2}{|c|}{ DIMENSIÓN 2: JUSTICLA SOCLLL Y EDUCACIÓN } & Relevancia & $\begin{array}{c}\text { Susceptibilida } \\
\text { d de cambio }\end{array}$ \\
\hline Indicador 1 & $\begin{array}{l}\text { Percepción personal del alumnado respecto a las } \\
\text { consecuencias de su esfuerzo ante las demandas } \\
\text { educativas. }\end{array}$ & .95 & .95 \\
\hline Indicador 2 & Percepción de su propia experiencia como educando. & .95 & .95 \\
\hline Indicador 3 & $\begin{array}{l}\text { Percepción de las familias y del alumnado acerca del modo } \\
\text { de acceso al éxito social y al bienestar. }\end{array}$ & .90 & .95 \\
\hline \multicolumn{2}{|c|}{ DIMENSIÓN 3: VALOR DIFERENCLAL DE LA EDUCACIÓN } & Relevancia & $\begin{array}{l}\text { Susceptibilida } \\
\text { d de cambio }\end{array}$ \\
\hline Indicador 1 & $\begin{array}{l}\text { Importancia de la educación para la vida: personal, laboral } \\
\text { y social. }\end{array}$ & 1 & 1 \\
\hline \multicolumn{2}{|c|}{ DIMENSIÓN 4: OBSTÁCULOS Y FACILITADORES } & Relevancia & $\begin{array}{l}\text { Susceptibilida } \\
\text { d de cambio }\end{array}$ \\
\hline Indicador 1 & $\begin{array}{l}\text { Percepción de los elementos o factores que pueden } \\
\text { favorecer o limitar el logro de los objetivos educativos } \\
\text { planteados tanto por el alumnado, como por sus familias y } \\
\text { el profesorado. }\end{array}$ & .95 & .95 \\
\hline
\end{tabular}

Nota: Adaptado de "La relevancia y susceptibilidad de cambio en la docencia del Valor Social Subjetivo de la Educación: validación de constructo”. En C. Sancho-Álvarez, J. Jornet, \& J. González-Such, 2014, Ponencia presentada en el $V$ Coloquio de la Red Iberoamericana de Investigadores sobre la Evaluación de la Docencia (RIIED). Ensenada, México: Universidad Autónoma de Baja California. 
Asimismo, se realiza un estudio de encuesta ${ }^{1}$ posterior sobre la advertencia de indicadores establecidos en función de dos criterios de valoración (relevancia y susceptibilidad de cambio) sobre dos audiencias diferenciadas (profesorado de escuelas -submuestra 1- y profesorado de universidades -submuestra 2-). Únicamente señalar que los criterios de valoración se refieren a la relevancia, en el sentido de la importancia que el indicador tiene para definir el constructo; y la susceptibilidad de cambio hace referencia al grado en que los expertos consideran que lo se mide a través de cada indicador es posible cambiarlo a través de una intervención psicosocio-educativa. Este segundo criterio estimamos que es necesario considerarlo desde una perspectiva de evaluación como instrumento para el cambio y la transformación personal y social.

Finalmente, después de los análisis de datos, se realiza un segundo comité de expertos/as para presentar una propuesta de constructo final fundamentada en indicadores de evaluación adecuados al proceso de enseñanza-aprendizaje.

\section{Comités de expertos/as}

Inicialmente se realiza un primer comité de expertos/as con docentes universitarios especializados en evaluación y medición educativas, para tratar de mejorar la formulación de los indicadores de evaluación sobre los datos de validación obtenidos en anteriores estudios de validación previa (Sancho-Álvarez et al., 2013).

El grupo de trabajo ${ }^{2}$ estuvo formado por 12 docentes de universidad (66,7\% mujeres; 33,3\% hombres), de edades comprendidas entre 25 y 55 años y con experiencia profesional en la Universidad -ver tabla 3-.

Tabla 3

Distribución de la muestra por experiencia docente universitaria

\begin{tabular}{llcc}
\hline \multirow{3}{*}{$\begin{array}{l}\text { Años totales de docencia } \\
\text { profesional }\end{array}$} & Frecuencia & Porcentaje \\
\cline { 2 - 4 } & Menos de 5 & 3 & $25 \%$ \\
& De 5 10 & 2 & $16.7 \%$ \\
& De 10 a 15 & 3 & $25 \%$ \\
& De 15 a 20 & 2 & $16.7 \%$ \\
& De 20 a 25 & 1 & $8.3 \%$ \\
& Más de 25 & 1 & $8.3 \%$ \\
\hline & & 12 & $100 \%$ \\
\hline
\end{tabular}

\section{Submuestra I}

Con el fin de estudiar la relevancia y susceptibilidad de cambio de cada uno de los indicadores propuestos anteriormente, se consulta a un grupo de docentes de

1 Encuesta online realizada con LimeSurvey para facilitar la administración y recogida de la información, adaptada al ámbito educativo por el grupo de innovación docente InnovaMide www.uv.es/innovamide

2 El comité de expertos/as final es constituido por las mismas personas que el comité inicial para evitar subjetividades ante las propuestas de mejora establecidas previamente. 
escuelas en relación a los dos criterios de valoración -relevancia y susceptibilidad de cambio- para obtener evidencias de validación según su percepción socio-educativa.

El grupo de personas respondientes estuvo formado por 20 docentes de escuelas (75\% mujeres; $25 \%$ hombres), de edades comprendidas entre 20 y 60 años (34 años de media), y con una experiencia profesional de entre menos de 5 años hasta más de 25 años en su centro escolar (en Infantil, Primaria o Secundaria).

Tabla 4

Distribución de la muestra por etapa escolar y por experiencia docente

\begin{tabular}{llcc}
\hline & & Frecuencia & Porcentaje \\
\cline { 2 - 4 } Etapa escolar en la que & Infantil & 3 & $15 \%$ \\
imparte actualmente su & Primaria & 10 & $50 \%$ \\
docencia & Secundaria & 7 & $35 \%$ \\
\hline Años totales de docencia & Menos de 5 & 6 & $30 \%$ \\
profesional & De 5 a 10 & 3 & $15 \%$ \\
& De 10 a 15 & 4 & $20 \%$ \\
& De 15 a 20 & 2 & $10 \%$ \\
& De 20 a 25 & 2 & $10 \%$ \\
& Más de 25 & 3 & $15 \%$ \\
\hline
\end{tabular}

\section{Submuestra 2}

Asimismo, se consulta también con posterioridad a un grupo de docentes universitarios en relación a estos dos criterios de valoración -relevancia y susceptibilidad de cambio- para obtener nuevas evidencias de validación desde otras perspectivas docentes.

El grupo de personas respondientes estuvo formado por 23 docentes de universidad (82,6\% mujeres; $17,4 \%$ hombres), de edades comprendidas entre 25 y 58 años (30 años de media), y con una experiencia profesional de entre menos de 5 años hasta más de 25 años en la Universidad.

Tabla 5

Distribución de la muestra por etapa escolar y experiencia docente

\begin{tabular}{llcc}
\hline \multirow{3}{*}{$\begin{array}{l}\text { Años totales de docencia } \\
\text { profesional }\end{array}$} & Frecuencia & Porcentaje \\
\cline { 2 - 4 } & Menos de 5 & 7 & $30.4 \%$ \\
& De 5 a 10 & 3 & $13.0 \%$ \\
& De 10 a 15 & 4 & $17.4 \%$ \\
& De 15 a 20 & 4 & $17.4 \%$ \\
& De 20 a 25 & 1 & $4.3 \%$ \\
& Más de 25 & 4 & $17.4 \%$ \\
\hline & & 23 & $100 \%$ \\
\hline
\end{tabular}




\section{Análisis de datos}

A partir de toda la información recogida, se han llevado a cabo diferentes análisis estadísticos sobre los datos para comprobar la concordancia inter-subjetiva de las valoraciones sobre cada escala:

- Análisis de la relevancia y susceptibilidad de cambio de los criterios de evaluación, a partir de las valoraciones emitidas por los jueces consultados; se procede a abordar y a presentar los datos mediante análisis estadísticos descriptivos a partir de los resultados extraídos.

- Consistencia inter-jueces; se ha analizado la fiabilidad de las valoraciones de los jueces, como garantía para establecer el grado de consenso inter-subjetivo, y en consecuencia, poder valorar la adecuación de las propuestas que se derivan de sus valoraciones -de relevancia y susceptibilidad de cambio-, mediante el análisis de correlación intra-clase y a través de la W Kendall para comprobar la concordancia y dispersión de las valoraciones en cada dimensión y aspecto evaluado (Kendall, 1963; Wallis, 1939).

- Validación cruzada entre muestras diferenciadas; se ha realizado un análisis por medio de la U Mann-Whitney seleccionando dos muestras diferenciadas mediante diferentes grupos de estudio -(1) profesorado de escuelas: Infantil, Primaria y Secundaria, y (2) profesorado de universidad- para determinar la estabilidad de los indicadores psicométricos o poder establecer diferencias (Barbero, Vila y Holgado, 2011; Mosier, 1951).

\section{Resultados}

\section{Comité de expertos/as inicial}

Después de recoger las diferentes propuestas de mejora en el equipo de trabajo inicial sobre el constructo previo, se advierten una serie de dimensiones para continuar el estudio sobre un constructo más fundamentado en indicadores consensuados, posterior a la primera validación realizada con anterioridad.

\section{Resultados submuestras}

A partir de los datos obtenidos sobre cada una de las submuestras -(1) profesorado de escuelas y (2) profesorado de universidades- se presentan a continuación ambos conjuntos de datos para cada una de las dimensiones teóricas trabajados sobre el constructo. Con el objetivo de observar de manera comparativa los resultados de las propias validaciones por jueces y poder llegar a obtener una validación final cruzada de cada una de ellas. A continuación, mediante gráficos, podemos observar el estudio completo en función de cada indicador de evaluación. 


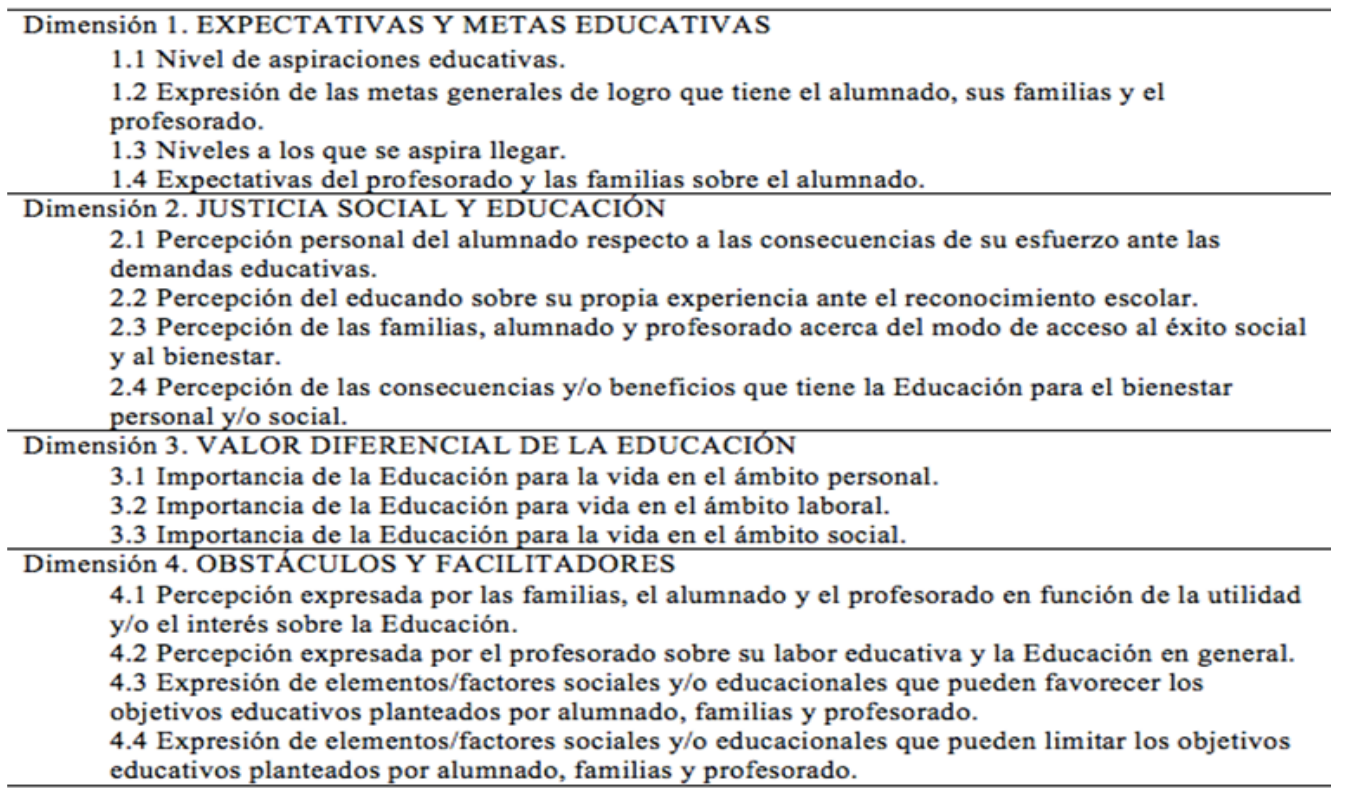

Cuadro 2. Propuesta de dimensiones e indicadores de evaluación para el VSE-Subjetivo

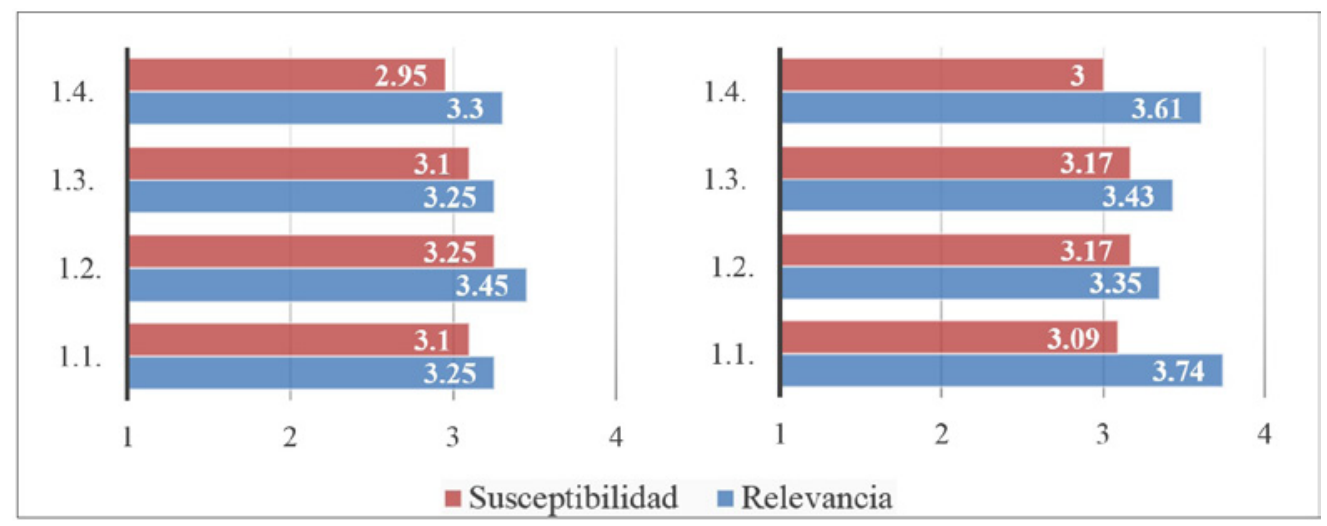

Gráfico 4. Comparativa entre submuestra 1 (profesorado escuela izquierda) y submuestra 2 (profesorado universidad derecha) sobre la dimensión 1.

Como podemos observar en el gráfico 4 las medias para cada indicador de la dimensión 1 (D1) son adecuadas, superando en la totalidad de los casos 2,95 en ambos criterios de evaluación -relevancia y susceptibilidad de cambio-, siendo la escala de respuesta de 1 a 4 puntos. Asimismo, para observar si el acuerdo es significativo entre las audiencias y comprobar las diferencias estadísticas en las valoraciones sobre la D1, podemos seguir la tabla que se presenta a continuación. 
Tabla 6

Concordancia del acuerdo inter-subjetivo entre validaciones sobre la D1

\begin{tabular}{lcllll}
\hline Dimensión & $N$ & $\begin{array}{l}\text { Relevancia } \\
\text { W de Kendall }\end{array}$ & $\begin{array}{l}\text { Sig. asintot. } \\
\text { (bilateral) }\end{array}$ & Susceptibilidad de cambio \\
1 val 1 & 20 & .607 & $.000^{*}$ & .716 & $\begin{array}{l}\text { Sig. asintot. } \\
\text { (bilateral) }\end{array}$ \\
1 val 2 & 23 & .285 & .294 & .672 & $.000^{*}$ \\
& & U Mann-Whitney & $\begin{array}{l}\text { Sig. asintot. } \\
\text { (bilateral) }\end{array}$ & U Mann-Whitney & $\begin{array}{l}\text { Sig. asintot. } \\
\text { (bilateral) }\end{array}$ \\
1 val 1,2 & 43 & 303.0 & .067 & 228.0 & .961 \\
\hline
\end{tabular}

\footnotetext{
*nivel de significancia .05
}

A partir de los datos obtenidos -ver tabla 6-, el acuerdo intersubjetivo del profesorado de escuela es significativo, como podemos observar a partir de los resultados de la prueba W de Kendall. Sin embargo, entre el profesorado de universidad el nivel de acuerdo es significativo únicamente en el criterio de susceptibilidad de cambio. En este sentido, cabe destacar que la diferencia no es estadísticamente significativa entre las dos audiencias de profesorado, lo que se observa a partir de los resultados de la prueba U Mann-Whitney para ambos criterios -relevancia y susceptibilidad de cambio-.

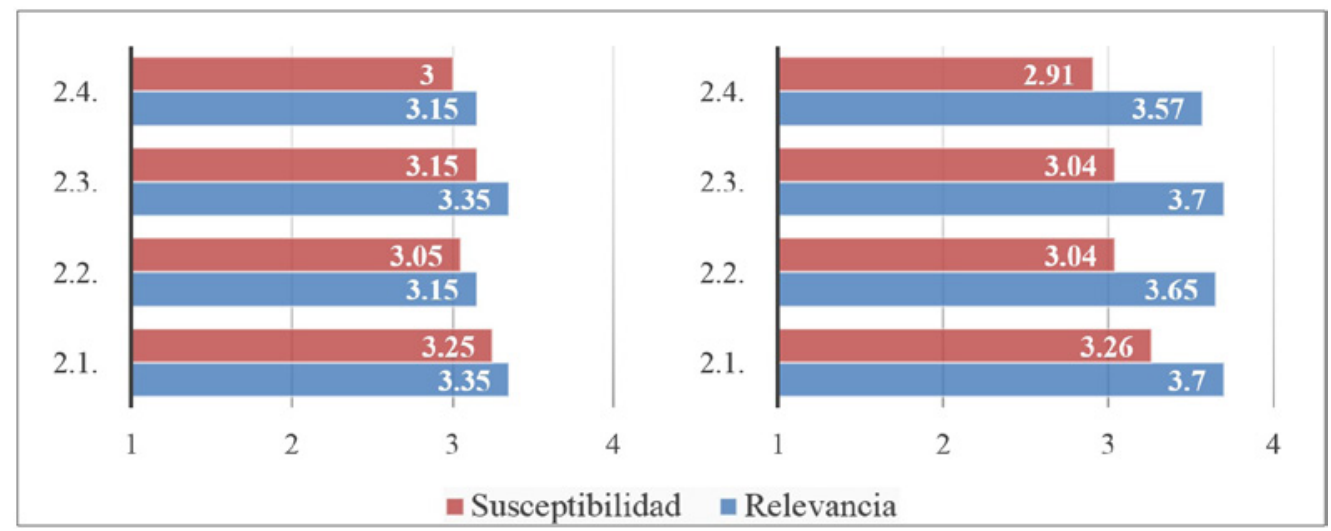

Gráfico 5. Comparativa entre submuestra 1 (profesorado escuela izquierda) y submuestra 2 (profesorado universidad derecha) sobre la dimensión 2.

En relación a la dimensión 2 (D2) observamos que, en todos los casos y en ambos criterios de valoración, las medias son superiores a 2,91 en su totalidad. En este sentido, a continuación podemos observar que el acuerdo es significativo para ambas audiencias y no presentan diferencias estadísticamente significativas. 
Tabla 7

Concordancia del acuerdo inter-subjetivo entre validaciones sobre la D2

\begin{tabular}{|c|c|c|c|c|c|}
\hline \multirow[b]{2}{*}{ Dimensión } & \multirow[b]{2}{*}{$N$} & \multicolumn{2}{|l|}{ Relevancia } & \multicolumn{2}{|c|}{ Susceptibilidad de cambio } \\
\hline & & $W$ de Kendall & $\begin{array}{l}\text { Sig. asintot. } \\
\text { (bilateral) }\end{array}$ & $W$ de Kendall & $\begin{array}{l}\text { Sig. asintot. } \\
\text { (bilateral) }\end{array}$ \\
\hline 2 val 1 & 20 & .572 & $.001 *$ & .601 & $.001 *$ \\
\hline \multirow[t]{2}{*}{2 val 2} & 23 & .485 & $.005 *$ & .666 & $.000 *$ \\
\hline & & U Mann-Whitney & $\begin{array}{l}\text { Sig. asintot. } \\
\text { (bilateral) }\end{array}$ & U Mann-Whitney & $\begin{array}{l}\text { Sig. asintot. } \\
\text { (bilateral) }\end{array}$ \\
\hline 1 val 1,2 & 43 & 338.5 & $.007 *$ & 213.0 & .675 \\
\hline
\end{tabular}

*nivel de significancia .05

En el gráfico que presentamos a continuación se observa que para la dimensión 3 (D3) las medias son menores que en las anteriores dos dimensiones, pero de todos los casos superan los 2,61 puntos. Por lo que, las valoraciones continúan presentando adecuación.

En este caso, para la D3 observamos que existe acuerdo significativo en cuanto a relevancia y susceptibilidad de cambio entre los dos grupos de profesorado. De todas formas, encontramos diferencias estadísticamente significativas entre grupos en el caso del criterio de relevancia.

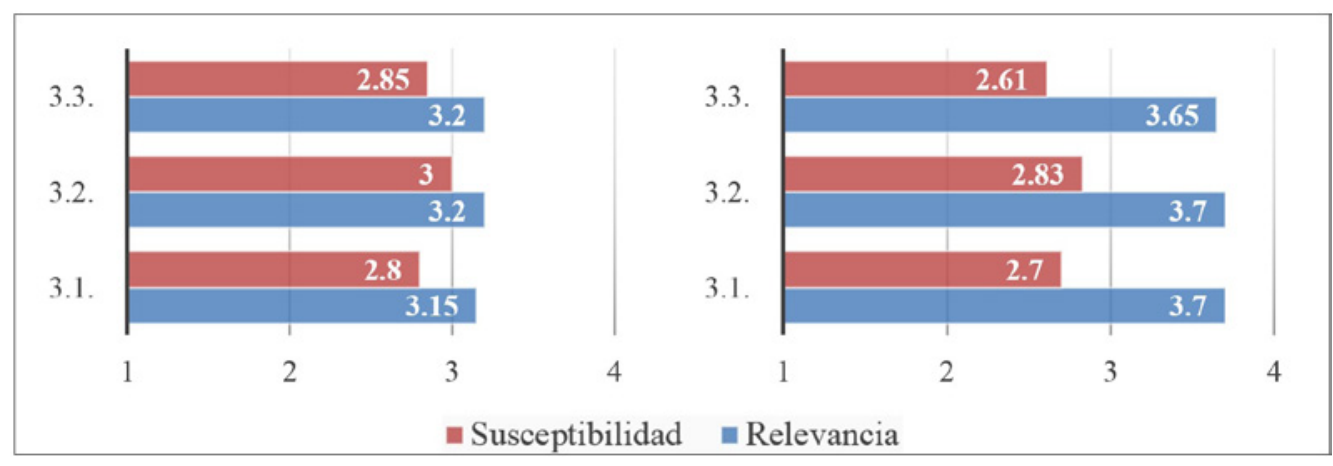

Gráfico 6. Comparativa entre submuestra 1 (profesorado escuela izquierda) y submuestra 2 (profesorado universidad derecha) sobre la dimensión 3.

Si hablamos de la dimensión 4 (D4), vemos como todas las medias de valoraciones siguen siendo elevadas -ver gráfico 7; todas superiores a 2,78 puntos-.

Se puede observar -ver tabla 4- que todas las valoraciones muestran un acuerdo significativamente elevado para cada uno de los criterios y audiencias. En esta línea, encontramos diferencias significativas para el caso de la relevancia entre los grupos de respondientes. Este aspecto deberá considerarse para reflexionar sobre el constructo en las conclusiones de este trabajo. 
Tabla 8

Concordancia del acuerdo inter-subjetivo entre validaciones sobre la D3

\begin{tabular}{llllll}
\hline Dimensión & $N$ & $\begin{array}{l}\text { Relevancia } \\
\text { W de Kendall }\end{array}$ & $\begin{array}{l}\text { Sig. asintot. } \\
\text { (bilateral) }\end{array}$ & Susceptibilidad de cambio \\
3 val 1 & 20 & .630 & $.011^{*}$ & .602 & $\begin{array}{l}\text { Sig. asintot. } \\
\text { (bilateral) }\end{array}$ \\
3 val 2 & 23 & .688 & $.002 *$ & .810 & $.017 *$ \\
& & U Mann-Whitney & $\begin{array}{l}\text { Sig. asintot. } \\
\text { (bilateral) }\end{array}$ & U Mann-Whitney & $\begin{array}{l}\text { Sig. asintot. } \\
\text { (bilateral) }\end{array}$ \\
1 val 1,2 & 43 & 339.5 & $.000^{*}$ & 193.5 & .367 \\
\hline
\end{tabular}

*nivel de significancia .05

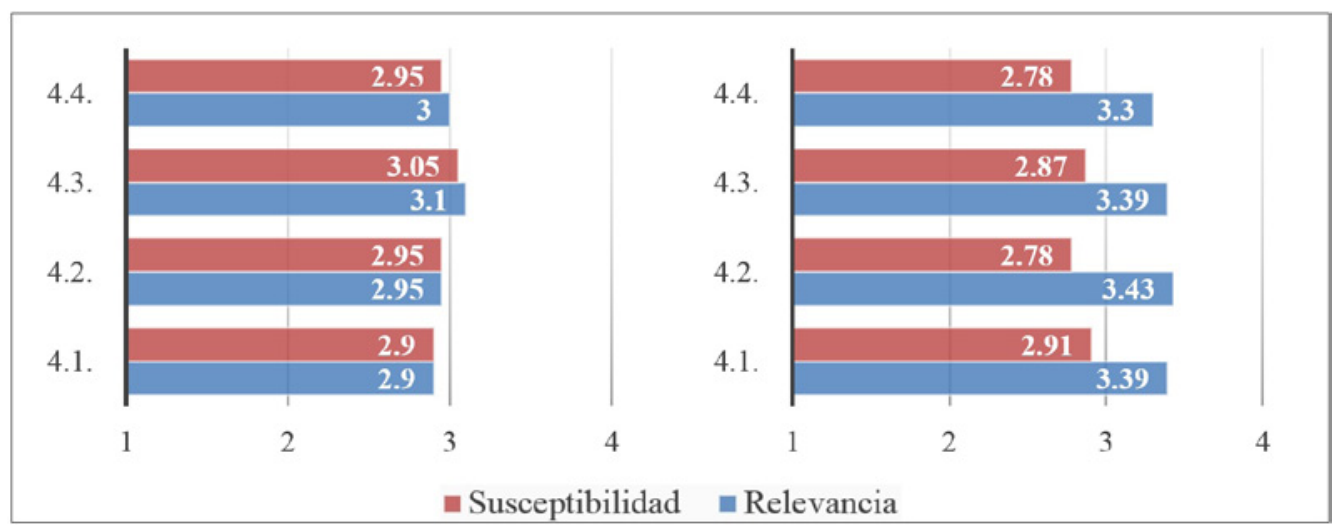

Gráfico 7. Comparativa entre submuestra 1 (profesorado escuela izquierda) y submuestra 2 (profesorado universidad derecha) sobre la dimensión 4.

Tabla 9

Concordancia del acuerdo inter-subjetivo entre validaciones sobre la D4

\begin{tabular}{llllll}
\hline Dimensión & $N$ & $\begin{array}{l}\text { Relevancia } \\
\text { W de Kendall }\end{array}$ & $\begin{array}{l}\text { Sig. asintot. } \\
\text { (bilateral) }\end{array}$ & Susceptibilidad de cambio \\
4 val 1 & 20 & .572 & $.001^{*}$ & .595 & $\begin{array}{l}\text { Sig. asintot. } \\
\text { (bilateral) }\end{array}$ \\
4 val 2 & 23 & .451 & $.012^{*}$ & .583 & $.001^{*}$ \\
& & U Mann-Whitney & $\begin{array}{l}\text { Sig. asintot. } \\
\text { (bilateral) }\end{array}$ & U Mann-Whitney & $\begin{array}{l}\text { Sig. asintot. } \\
\text { (bilateral) }\end{array}$ \\
1 val 1,2 & 43 & 366.0 & $.000^{*}$ & 179.0 & .196 \\
\hline
\end{tabular}

*nivel de significancia .05 


\section{Discusión y conclusiones}

En general, si observamos los datos de manera sintética en relación a la significación del acuerdo intersubjetivo estudiado sobre el propio constructo, encontramos una fundamentación sólida entre todas las dimensiones. En este sentido, el estudio realizado de validación cruzada avala el constructo Valor Social Subjetivo de la Educación presentado por indicadores de evaluación -ver cuadro 3-.

Cabe destacar una limitación importante ante el trabajo que desarrollamos, ya que hubiera sido muy interesante haber constituido los comités de expertos/as de manera presencial también con profesorado de escuela, al igual que se ha realizado con el profesorado de universidad; ya que, después de observar los datos, hubiera sido relevante intentar buscar aquellas diferencias significativas obtenidas entre grupos de respondientes, que han resultado para cada uno de los criterios y dimensiones. Algo que sin duda, se continuará trabajando en futuras investigaciones.

\section{Dimensión 1. EXPECTATIVAS Y METAS EDUCATIVAS}

\begin{tabular}{|c|c|}
\hline Indicador 1 & Nivel de aspiraciones educativas \\
\hline Indicador 2 & $\begin{array}{l}\text { Expresión de las metas generales de logro que tiene el alumnado, sus familias y el } \\
\text { profesorado }\end{array}$ \\
\hline Indicador 3 & $\begin{array}{l}\text { Expectativas sobre el alumnado según la situación socioeconómica y cultural de la } \\
\text { familia }\end{array}$ \\
\hline Indicador 4 & Expectativas del profesorado y las familias sobre el logro esperado del alumnado \\
\hline
\end{tabular}

\section{Dimensión 2. JUSTICIA SOCIAL Y EDUCACIÓN}

\begin{tabular}{|c|c|}
\hline Indicador 1 & $\begin{array}{l}\text { Percepción personal del alumnado respecto a las consecuencias de su esfuerzo ante las } \\
\text { demandas educativas }\end{array}$ \\
\hline Indicador 2 & Percepción del educando sobre su propia experiencia ante el reconocimiento escolar \\
\hline Indicador 3 & $\begin{array}{l}\text { Percepción de las familias, alumnado y profesorado acerca del modo de acceso al éxito } \\
\text { social y al bienestar }\end{array}$ \\
\hline Indicador 4 & $\begin{array}{l}\text { Percepción de las consecuencias y/o beneficios que tiene la Educación para el bienestar } \\
\text { personal y/o social }\end{array}$ \\
\hline \multicolumn{2}{|c|}{ Dimensión 3. VALOR DIFERENCIAL DE LA EDUCACIÓN } \\
\hline $\begin{array}{l}\text { Indicador } 1 \\
\text { Indicador } 2 \\
\text { Indicador } 3 \\
\end{array}$ & $\begin{array}{l}\text { Importancia de la Educación para la vida en el ámbito personal } \\
\text { Importancia de la Educación para la vida en el ámbito laboral } \\
\text { Importancia de la Educación para la vida en el ámbito social }\end{array}$ \\
\hline \multicolumn{2}{|c|}{ Dimensión 4. OBSTÁCULOS Y FACILITADORES } \\
\hline $\begin{array}{l}\text { Indicador } 1 \\
\text { Indicador } 2\end{array}$ & $\begin{array}{l}\text { Percepción de factores sociales que favorecen una visión positiva de la Educación } \\
\text { Percepción de factores sociales que limitan una visión positiva de la Educación }\end{array}$ \\
\hline
\end{tabular}

Cuadro 3. Propuesta de dimensiones e indicadores de evaluación para el Valor Social Subjetivo de la Educación 
A partir de todos los comentarios y propuestas de mejora recogidas en el comité de expertos/as final, advertimos una serie de indicadores de evaluación para cada una de las dimensiones sobre el constructo trabajado de Valor Social Subjetivo de la Educación; lo que nos conduce a realizar alguna modificación sobre la formulación de los indicadores en función de los datos obtenidos sobre las validaciones de manera cuantitativa y con respecto a las propuestas de mejora del grupo de trabajo de manera cualitativa.

La educación superior favorece la cohesión social y la participación y consolidación de los valores democráticos, la reducción de la marginalidad y la delincuencia además de la garantía de una mejor educación de las futuras generaciones que podrán acceder a más recursos (Santín, 2002, p. 5).

De acuerdo a la anterior definición podemos comprender que desde determinadas autorías se percibe un adecuado VSE desde, en este caso, la etapa de profesorado universitario. En nuestro caso, al haber estudiado este término con la participación de dos audiencias diferenciadas (profesorado de escuela y universidad) podemos observar claramente que ese valor educativo no es el mismo para cada uno de ellos/ as. Ya que, a partir de los resultados -ver punto resultados submuestras- la diferencia de las valoraciones entre audiencias es significativa en las dimensiones 2, 3 y 4 para la escala de relevancia; donde podemos observar que se encontrarían indicadores en relación a temas tales como la justicia social y educación, el valor diferencial de la educación u obstáculos y facilitadores hacia la propia visión positiva de la educación. Por lo que, estos datos muestran que la percepción entre profesorado de escuelas -infantil, primaria y secundaria- y universidad no es igual ante esta serie de tópicos, ya que pueden tener visiones diferenciadas dependiendo de su situación en la etapa escolar del sistema educativo donde se enmarcan. Algo que, sin duda, debe ser analizado en profundidad en estudios posteriores para intentar desvelar aquellas claves socioeducativas que puedan estar influyendo al respecto. Por lo que, si observamos estudios recientes, estas diferencias pueden presentarse, en relación al colectivo de profesorado universitario, debido a sus expectativas y concepciones particulares (López, Pérez-García y Rodríguez, 2015), o incluso, de acuerdo a otras líneas investigativas, debido a la formación y la experiencia docente del profesorado, que influyen o pueden influir en el desarrollo de actitudes favorables hacia la propia práctica docente (Ocampo y Cid, 2012).

En esta línea de trabajo, se proponen como líneas futuras de actuación la propuesta de un cuestionario piloto dirigido a la Educación Secundaria para estudiar y valorar el constructo de VSE-Subjetivo en este ámbito. Asimismo, para poder establecer un lineamiento sólido de investigación, la propuesta debe estar valorada por profesionales de la etapa educativa en cuestión, para poder mejorar el propio cuestionario antes de su administración sobre el alumnado.

Por ello, debemos de ser conscientes de la repercusión del VSE-Subjetivo durante el proceso de enseñanza-aprendizaje entre el profesorado, ya que dependiendo de la percepción de cada colectivo en cada etapa educativa, como hemos visto anteriormente, podemos estar brindando una práctica educativa diferenciadora, al no creer en la uti- 
lidad y valor de la educación desde nuestra labor docente, incluso llegar a transmitir estos valores subjetivos -adecuados o inadecuados sobre el hecho educativo- hacia el alumnado (Montalvà. 2013).

El tópico del que se habla con frecuencia entre las familias y la sociedad en relación a cómo valoran al profesorado, sin duda, es una opinión conjunta que se crea desde la sociedad hacia este tipo profesionales, y que puede ser diferente según el contexto donde nos situemos. Así como se ha constatado que el nivel sociocultural de las familias condiciona, por ejemplo, la diferente percepción que de las prácticas educativas llegan a tener padres y madres (Alonso y Román, 2014). Algo que si se observa de manera más específica, a partir de un estudio realizado por el Centro de Investigaciones Sociológicas (CIS, 2013) para recoger información sobre "qué valoración le merecen una serie de profesiones u oficios", se concluye que el nivel de prestigio profesional varía en función de la etapa escolar dependiendo donde se sitúe el docente (maestros/as E. Infantil Med 74.64 DT 24.06; profesor/a primaria Med 74.70 DT 23.71; profesor/a secundaria Med 73.67 DT 23.91; profesor/a formación profesional Med 73.92 DT 23.08; profesor/a universidad Med 75.16 DT 23.58;). Este aspecto se relaciona directamente con el VSE-Subjetivo dependiendo de la valoración de los agentes en cada nivel educativo y los beneficios percibidos por la sociedad sobre la propia formación educativa en cada sector analizado (Jornet et al., 2011). "Sin lugar a dudas, la influencia educativa de los maestros se convierte en una piedra angular para iniciar el cambio hacia otra conciencia social" (Molina y Nunes, 2012, p. 383).

Por ejemplo, algunos factores pueden asociarse a una mejor valoración del profesorado universitario, ya sea por la función social que realiza la universidad (Pardo, 2011) o incluso por el impacto de la investigación educativa en la propia práctica docente universitaria (Díaz, Fernández-Cano, Faouzi y Henríquez, 2015). En definitiva, de acuerdo a la investigación actual, el papel del profesorado hoy en día, a partir de su función docente y social, juega un rol fundamental en la construcción del valor escolar entre los discentes (Prieto, 2008), así como se desarrolla una determinada imagen social docente (Zamora y Cabrera, 2015).

Todo esto nos lleva a plantearnos la importancia suma que posee el papel del profesorado en un entorno escolar, así como la responsabilidad máxima que tiene a la hora de establecer las diferentes relaciones que se producen en el binomio alumno-profesor dentro del aula. El docente va a sumar dentro de su práctica educativa diferentes creencias, convicciones y escalas de valores que, sin lugar a dudas, va a transmitir en su discurso pedagógico con sus alumnos (Prieto, 2008, p. 328).

\section{Referencias}

Alonso, J. \& Román, J. M. (2014). Nivel sociocultural, prácticas educativas familiares y autoestima de los hijos en edades tempranas. Revista de Investigación Educativa, 32(1), 187-202. doi: http://dx.doi.org/10.6018/rie.32.1.173421

Backhoff, E., Bouzas, A., González-Montesinos, M., Andrade, E., Hernández, E., \& Contreras, C. (2008). Factores asociados al aprendizaje de estudiantes de 3o de primaria en México (1 $1^{\underline{a}} e$ d.). México D.F.: Instituto Nacional para la Evaluación de la Educación (INEE). 
Baer, J. (1999). The effects of family structure and SES on family processes in early adolescence. Journal of Adolescence, 22(3), 341-54.

Barbero, M. I., Vila, E., \& Holgado, F. P. (2011). Introducción básica al análisis factorial. Madid: UNED.

Bericat, E. (1998). La integración de los métodos cuantitativo y cualitativo en la investigación social. Significado y medida. Barcelona: Ariel.

Carstensen, C., Rost, J., \& Prenzel, M. (2003). Proposal for assesing the Affective Domain. Las Vegas: Expert Group Meeting.

Casanova, J. (15 de septiembre de 2011). El valor de la educación. El País. Recuperado de http://elpais.com/diario/2011/09/15/opinion/1316037605_850215.html

Centro de Investigaciones Sociológicas [CIS] (2013). Estudio 2978. Barómetro de febrero de 2013. Madrid: CIS.

Coleman, J. S. et al., (1966). Equality of educational opportunity (2 vols.).Washington, DC: Government Printing Office

Cronbach, L. J. (1988). Five perspectives on validity argument. En H. Wainer \& H. Braun (Eds.), Test validity (pp. 3-17). Hillsdale, NJ: Lawrence Erlbaum.

De la Orden, A., \& Jornet, J. M. (2012). La utilidad de las evaluaciones de sistemas educativos: el valor de la consideración del contexto. Revista de Pedagogía Bordón, 64(2), 69-88.

De la Orden, A. (2007). Evaluación de la calidad de la educación. Un modelo sistémico como base para la construcción de un sistema de indicadores. México: Instituto Nacional para la Evaluación de la Educación (INEE).

Delors, J. (1996). La educación encierra un tesoro. Madrid: Santillana - UNESCO.

Díaz, E., Fernández-Cano, A., Faouzi, T., \& Henríquez, C. F. (2015). Validación del constructo subyacente en una escala de evaluación del impacto de la investigación educativa sobre la práctica docente mediante análisis factorial confirmatorio. Revista de Investigación Educativa, 33(1), 47-63. doi: http://dx.doi.org/10.6018/rie.33.1.193521

García, A. (2012). El valor de la Educación. Recuperado de https://www.yoestudieenlapublica.org/aula.php

García-García, M., Biencinto-López, C., Carpintero-Molina, E., Núñez-del-Río, M. C., \& Arteaga-Martínez, B. (2013). Rendimiento en matemáticas y actitud hacia la materia en centros inclusivos: estudio en la Comunidad de Madrid. Revista de Investigación Educativa, 31(1), 117-132. doi: http://dx.doi.org/10.6018/rie.31.1.143221

Gil, J. (2014). Factores asociados a la brecha regional del rendimiento español en la evaluación pisa. Revista de Investigación Educativa, 32(2), 393-410. doi: http://dx.doi. org/10.6018/rie.32.2.192441

Glewwe, P., \& Kremer, M. (2006). Schools, Teachers, and Education Outcomes in Developing Countries. En Hanushek, E., \& Welch,F. (Eds.). Handbook of the economics of education (vol. 2, pp. 945-1017). New York, NY: Elsevier.

Herrera, M. (2009). El valor de la escuela y el fracaso escolar. Revista Iberoamericana sobre Calidad, Eficacia y Cambio en Educación, 7(4), 254-263. Recuperado de http:// www.redalyc.org/articulo.oa?id=55114094013

Jornert, J. M. (2012). Dimensiones Docentes y Cohesión Social: Reflexiones desde la Evaluación. Revista Iberoamericana de Evaluación Educativa, 5(1e), 349-362. http://www. rinace.net/riee/numeros/vol5-num1_e/art27.pdf 
Jornet, J. M. (2010). Dimensiones docentes y cohesión social: reflexiones desde la evaluación. Ponencia presentada en el II Coloquio de la Red Iberoamericana de Investigación sobre la docencia (RIIED). Valencia: Universitat de València.

Jornet, J. M. (1989). Proyecto docente para el acceso a Profesores Titulares de Universidad. Perfil: Medición y Evaluación Educativas. Valencia: Universitat de València. Manuscrito no publicado. Bordón, Revista de Pedagogía, 64(2), 89-110.

Jornet, J. M., \& González-Such, J. (2009). Evaluación criterial: determinación de estándares de interpretación (EE) para pruebas de rendimiento educativo. Estudios sobre Educación, 16, 103-123.

Jornet, J. M., González-Such, J., \& Perales, Ma J. (2013). Investigación evaluativa: Una perspectiva basada en la complementariedad metodológica (cuantitativa-cualitativa). Perú: CREA.

Jornet, J. M., González-Such, J., \& Perales, Ma․ J. (2012). Diseño de cuestionarios de contexto para la evaluación de sistemas educativos: optimización de la medida de constructos complejos. (2012). Jornet, J. M., Perales, M. J., \& Sánchez-Delgado, P. (2011). El Valor Social de la Educación: Entre la Subjetividad y la Objetividad. Consideraciones Teórico-Metodológicas para su Evaluación. Revista Iberoamericana de Evaluación Educativa, 4(1), 51-77. Recuperado de http://www.rinace.net/riee/ numeros/vol4-num1/art3.pdf

Jornet, J. M., Sánchez-Delgado, P., \& Perales, M. J. (2014). La evaluación del impacto y la relevancia de la educación en la sociedad. Valencia: PUV.

Jornet, J. M., Sancho-Álvarez, C., \& Sánchez-Delgado, P. (2014). Una aproximación, mediante juicio, a la validación del Modelo de Evaluación para el desarrollo de la Cohesión Social (CS) a partir de la Educación. Ponencia presentada en el V Coloquio de la Red Iberoamericana de Investigadores sobre la Evaluación de la Docencia (RIIED). Ensenada, México: Universidad Autónoma de Baja California.

Jornet, J. M., \& Suárez, J.M. (1996). Pruebas estandarizadas y evaluación del rendimiento: usos y características métricas. Revista de Investigación Educativa, 14(2), 141-163.

Kane, M. T. (2001). Current Concerns in Validity Theory. Journal of Educational Measurement, 38(4), 319-342.

Kendall, M. G. (1963). Rank correlation methods. Londres: Griffin.

Krüger, N., Formichella, M. M., \& Lekuona, A. (2015). Más allá de los logros cognitivos: la actitud hacia la escuela y sus determinantes en España según PISA 2009. [Beyond cognitive-skills: the attitude towards school and its determinants in Spain with PISA 2009]. Revista de Educación, 367, 10-35. doi: 10.4438/1988-592X-RE-2015-367-281

López, M. C., Pérez-García, M. P., \& Rodríguez, M. J. (2015). Concepciones del profesorado universitario sobre la formación en el marco del espacio europeo de educación superior. Revista de Investigación Educativa, 33(1), 179-194. doi: http://dx.doi. org/10.6018/rie.33.1.189811

Mérida, R., Serrano, A., \& Tabernero, C. (2015). Diseño y validación de un cuestionario para la evaluación de la autoestima en la infancia. Revista de Investigación Educativa, 33(1), 149-162. doi: http://dx.doi.org/10.6018/rie.33.1.182391

Ministerio de Educación. (2011). Panorama de la educación Indicadores de la OCDE 2011. INFORME ESPAÑOL. Madrid: Instituto de Evaluación. Recuperado de http://www.mecd.gob.es/dctm/ievaluacion/indicadores-educativos/panorama-2011. pdf?documentId=0901e72b80eb8ea5 
Molina, J., \& Nunes, R. M. (2012). La percepción social de los futuros maestros sobre las personas con síndrome de Down: aplicación de la escala EPSD-1. Revista de Investigación Educativa, 30(2), 383-396. doi: http://dx.doi.org/10.6018/rie.30.2.130841

Montalvà, E. (2013). Valor social subjetivo de la educación para el profesorado: bases para la construcción de un instrumento de medida. Valencia: RODERIC. Recuperado de http:// roderic.uv.es/bitstream/handle/10550/35921/VSSE.pdf? sequence=1\&isAllowed=y

Mosier, C. (1951) Problems and designs of cross-validation. Educational and Psychological Measurement, 11, 5-11. doi: 10.1177/001316445101100101

Ocampo, C. I., \& Cid, B. (2012). Formación, experiencia docente y actitudes de los profesores de infantil y primaria ante la educación escolar de hijos de personas inmigrantes en España. Revista de Investigación Educativa, 30(1), 111-130. doi: http:// dx.doi.org/10.6018/rie.30.1.114321

Organización para la Cooperación y el Desarrollo Económicos [OCDE] (2006). Informe PISA 2006. Competencias científicas para el mundo del mañana. París: OCDE.

Organización para la Cooperación y el Desarrollo Económicos [OCDE] (2008). Informe PISA 2006. Competencias científicas para el mundo del mañana. Madrid: Santillana.

Organización para la Cooperación y el Desarrollo Económicos [OCDE] (2010). Regards sur l'éducation 2010. Les indicateurs de l'OCDE. París: OCDE.

Organización para la Cooperación y el Desarrollo Económicos [OCDE] (2011). Education at a Glance 2011: OECD indicators. Paris: OCDE.

Organización para la Cooperación y el Desarrollo Económicos [OCDE] (2012). Education at a Glance 2012: OECD indicators. Paris: OCDE.

Organización para la Cooperación y el Desarrollo Económicos [OCDE] (2013). Education at a Glance 2013: OECD indicators. Paris: OCDE.

Organización para la Cooperación y el Desarrollo Económicos [OCDE] (2014). Education at a Glance 2014: OECD indicators. Paris: OCDE.

Pardo, L. P. (2011). La función de la universidad en las sociedades del conocimiento. Aula: Revista de Pedagogía de la Universidad de Salamanca, 17, 145-158.

Prieto, E. (2008). El papel del profesorado en la actualidad. Su función docente y social. Foro de Educación, 10, 325-345. Recuperado de http://www.forodeeducacion.com/ numero10/020.pdf

Ruiz, G. (2009). La calidad del sistema educativo mexicano desde los resultados de evaluaciones nacionales. El aprendizaje en matemáticas. Revista Iberoamericana de Evaluación Educativa, 2(1), 74-89.

Sancho-Álvarez, C. (2013). Validación de constructo de un instrumento para evaluar el valor social subjetivo de la educación en la comunidad educativa. Valencia: Universitat de València: RODERIC. Recuperado de http://roderic.uv.es/bitstream/handle/10550/36216/ Sancho-\%c3\%81lvarez_2013.

Sancho-Álvarez, C., Jornet, J., \& Perales, M. J. (2013). Valor Social Subjetivo de la Educación: docencia y resultados escolares. Revista Iberoamericana de Evaluación Educativa, 7(2e), 197-207. Recuperado de http://www.rinace.net/riee/numeros/vol7num2e/art15.pdf

Sancho-Álvarez, C., Jornet, J., \& González-Such, J. (2014). La relevancia y susceptibilidad de cambio en la docencia del Valor Social Subjetivo de la Educación: validación de constructo. Ponencia presentada en el $V$ Coloquio de la Red Iberoamericana de Inves- 
tigadores sobre la Evaluación de la Docencia (RIIED). Ensenada, México: Universidad Autónoma de Baja California.

Santín, D. (2003). Descentralización y financiación de la Universidad Pública en Latinoamérica: Algunas propuestas a partir del caso español. Documento de trabajo de la Facultad de Ciencias Económicas y Empresariales, 1.

Soriano, E. (2011). El valor de la educación en un mundo globalizado. Madrid: Editorial La Muralla.

Wallis, W. A. (1939). The correlation ratio for ranked data. Journal of the American Statistical Association, 34, 533-538.

Willms, J. D. (2006). Learning Divides: Ten Policy Questions About the Performance and Equity of Schools and Schooling Systems. Montreal: UNESCO.

Willms, J. D., \& Somers, M. A. (2001). Family, Classrooms, and School Effects on Children's Educational Outcomes in Latin America. School Effectiveness and School Improvement, 12(4), 409-445.

Zamora, B. \& Cabrera, L. (2015). La sociedad y el profesorado. Imágenes y opiniones sociales sobre el profesorado. Revista de la Asociación de Sociología de la Educación, $8(1), 86-107$.

Fecha de recepción: 30 de abril de 2015.

Fecha de revisión: 30 de abril de 2015.

Fecha de aceptación: 29 de marzo de 2016. 